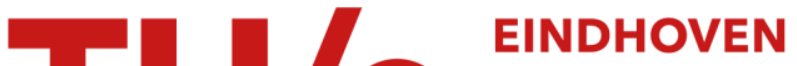 UNIVERSITY OF TECHNOLOGY
}

\section{Bunch self-focusing regime of laser wakefield acceleration with reduced emittance growth}

\section{Citation for published version (APA):}

Reitsma, A. J. W., Goloviznin, V. V., Kamp, L. P. J., \& Schep, T. J. (2002). Bunch self-focusing regime of laser wakefield acceleration with reduced emittance growth. Physical Review Letters, 88(1), 014802-1/4. [014802]. https://doi.org/10.1103/PhysRevLett.88.014802

DOI:

10.1103/PhysRevLett.88.014802

Document status and date:

Published: 01/01/2002

\section{Document Version:}

Publisher's PDF, also known as Version of Record (includes final page, issue and volume numbers)

\section{Please check the document version of this publication:}

- A submitted manuscript is the version of the article upon submission and before peer-review. There can be important differences between the submitted version and the official published version of record. People interested in the research are advised to contact the author for the final version of the publication, or visit the $\mathrm{DOI}$ to the publisher's website.

- The final author version and the galley proof are versions of the publication after peer review.

- The final published version features the final layout of the paper including the volume, issue and page numbers.

Link to publication

\section{General rights}

Copyright and moral rights for the publications made accessible in the public portal are retained by the authors and/or other copyright owners and it is a condition of accessing publications that users recognise and abide by the legal requirements associated with these rights.

- Users may download and print one copy of any publication from the public portal for the purpose of private study or research.

- You may not further distribute the material or use it for any profit-making activity or commercial gain

- You may freely distribute the URL identifying the publication in the public portal.

If the publication is distributed under the terms of Article 25fa of the Dutch Copyright Act, indicated by the "Taverne" license above, please follow below link for the End User Agreement:

www.tue.nl/taverne

Take down policy

If you believe that this document breaches copyright please contact us at:

openaccess@tue.nl

providing details and we will investigate your claim. 


\title{
Bunch Self-Focusing Regime of Laser Wakefield Acceleration with Reduced Emittance Growth
}

\author{
A. J. W. Reitsma, ${ }^{1}$ V. V. Goloviznin, ${ }^{1}$ L. P. J. Kamp, ${ }^{1}$ and T. J. Schep ${ }^{2}$ \\ ${ }^{1}$ Technische Universiteit Eindhoven, P.O. Box 513, 5600 MB Eindhoven, The Netherlands \\ ${ }^{2}$ FOM Instituut voor Plasmafysica Rijnhuizen, P.O. Box 1207, 3430 BE Nieuwegein, The Netherlands
}

(Received 22 June 2001; published 19 December 2001)

A new regime of laser wakefield acceleration of an injected electron bunch is described. In this regime, the bunch charge is so high that the bunch wakefields play an important role in the bunch dynamics. In particular, the transverse bunch wakefield induces a strong self-focusing that suppresses the transverse emittance growth arising from misalignment errors. The decelerating longitudinal bunch wakefield, however, is not so strong that it completely cancels the accelerating laser wakefield. In fact, the induced energy spread can be compensated by exploiting phase slippage effects. These features make the new regime interesting for high beam quality laser wakefield acceleration.

DOI: 10.1103/PhysRevLett.88.014802

In the past few years, laser wakefield acceleration [1] in the self-modulated regime has been demonstrated successfully in several proof-of-principle experiments [2,3]. Accelerating electric fields up to a few $100 \mathrm{GV} / \mathrm{m}$ and jets of accelerated electrons have been observed. In these experiments, the electrons are extracted from the bulk plasma by nonlinear processes like wave breaking or Raman forward scattering, which makes it hard to control the quality of the extracted electron bunch. Controlled acceleration is generally assumed to be possible in the resonant regime with external injection of the electrons.

In recent papers, control of energy spread [4-6] and of transverse emittance degradation [7-11] have been investigated. For the energy spread, the influence of the longitudinal bunch wakefield is important. Since the bunch wakefield is usually decelerating, there is a maximum charge that can be accelerated on a given laser wakefield: this is the well-known beam loading limit [4]. The energy spread induced by the bunch wakefield can be compensated by phase slippage effects [5]: this requires a proper tuning of injection phase and bunch length [6].

For transverse emittance degradation, two important sources have been found: mismatch between the particle distribution and the betatron orbits [7,8] and injection errors arising from misalignment or noise in the system $[9,10]$. In the presence of a strong bunch wakefield, injection errors are potentially more dangerous: the bunch wakefield introduces a coupling of the transverse oscillations at different axial positions inside the bunch, resulting in beam breakup instability [11].

Therefore, one expects that an increase of bunch charge, which gives a stronger bunch wakefield, leads to enhanced emittance growth. However, above a certain threshold this trend reverses and a high bunch charge regime with reduced emittance growth exists. We have found this regime by simulating acceleration of electron bunches injected with a certain radial offset from the propagation axis (see Fig. 1), and by varying the strength of the bunch wakefields. A simple model for the transverse bunch dynamics is presented to explain the reduction of emittance growth
PACS numbers: 41.75.Jv, 29.27.Fh, 52.40.Mj, 52.75.Di

as a consequence of self-focusing of the electron bunch [12]. Our simulation results show that the indicated charge threshold is below the beam loading limit.

For the description of the wakefields, the linear hydrodynamic equations for electron plasma waves in a homogeneous plasma are used. The quasistatic approximation is applied, so that the wakefields depend on the transverse coordinates $\vec{r}_{\perp}=(x, y)$ and the phase $\zeta=z-v_{\varphi} t$, where $z$ is the longitudinal coordinate, $v_{\varphi}$ is the phase velocity of the plasma wave (close to $c$, with Lorentz factor $\gamma_{\varphi} \gg 1$ ), and $t$ denotes the time. It is well known [13] that the wakefields can be derived from a wakefield potential $\Phi$, which is the difference between the electrostatic potential and the axial component of the vector potential. The wakefield potential is calculated from the (dimensionless) envelope of the laser pulse vector potential $a_{0}\left(\vec{r}_{\perp}, \zeta\right)$ and the electron bunch density $n_{b}\left(\vec{r}_{\perp}, \zeta\right)$ using the wakefield equation

$$
\left(k_{p}^{2}+\frac{\partial^{2}}{\partial \zeta^{2}}\right)\left(k_{p}^{2}-\nabla_{\perp}^{2}\right) \Phi=k_{p}^{2}\left(k_{p}^{2}-\nabla_{\perp}^{2}\right) \frac{a_{0}^{2}}{4}-k_{p}^{4} \frac{n_{b}}{n_{p}},
$$

where $n_{p}$ denotes the plasma density and $k_{p}$ is the plasma wave number, defined by $k_{p}^{2}=4 \pi n_{p} e^{2} / m c^{2}$. The wakefield potential is written as the sum of the laser wakefield potential $\Phi_{L}\left(\vec{r}_{\perp}, \zeta\right)$ and the bunch wakefield potential $\Phi_{B}\left(\vec{r}_{\perp}, \zeta\right)$, which are convolution integrals of

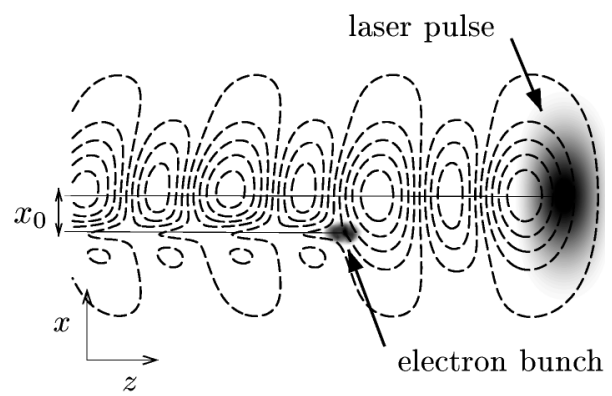

FIG. 1. Sketch of the laser pulse and the electron bunch with radial offset $x_{0}$ and contours of constant $\Phi$. 
$a_{0}$ and $n_{b} / n_{p}$, respectively, with the appropriate Green's functions. We assume that the laser pulse evolves slowly on the time scales of interest, so that we can prescribe $a_{0}$, and also $\Phi_{L}$, as a function of $\vec{r}_{\perp}$ and $\zeta$. The bunch density $n_{b}$, and therefore also $\Phi_{B}$, is implicitly time dependent because of the bunch dynamics, which follows from the equations of motion

$$
\frac{d \vec{P}}{d t}=-m c^{2} \nabla \Phi, \quad \frac{d \vec{r}_{\perp}}{d t}=\vec{v}_{\perp}, \quad \frac{d \zeta}{d t}=v_{z}-v_{\varphi}
$$

These equations describe the motion of a relativistic electron with momentum $\vec{P}$ (velocity $\vec{v}$ ) and coordinate $\left(\vec{r}_{\perp}, \zeta\right.$ ). The gradient $\nabla$ stands for $\left(\nabla_{\perp}, \partial / \partial \zeta\right)$.

In our simulations we use $2 \mathrm{D}$ slab geometry with $x$ as the transverse coordinate. The laser wakefield potential is

$$
\Phi_{L}(x, \zeta)=\Phi_{0} \sin \left(k_{p} \zeta\right) e^{-x^{2} / r_{L}^{2}},
$$

where $\Phi_{0}$ is the overall amplitude of the wakefield and $r_{L}$ is the spot size of the laser pulse. For simulation results presented in this paper, we have chosen $\Phi_{0}=0.1$, $k_{p} r_{L}=5$, and $\gamma_{\varphi}=70$, which corresponds to a $35 \mathrm{TW}$ laser pulse with $r_{L}=45 \mu \mathrm{m}$ in a plasma with density $3.5 \times 10^{17} \mathrm{~cm}^{-3}$. The electron bunch is represented by $N$ simulation particles, with coordinates $\left(x_{i}, \zeta_{i}\right)$. For the bunch density distribution of simulation particles we take $\delta$ functions:

$$
\frac{n_{b}}{n_{p}}(x, \zeta)=2 \Phi_{0} \frac{\eta}{N} \sum_{i=1}^{N} \delta\left(k_{p} x-k_{p} x_{i}\right) \delta\left(k_{p} \zeta-k_{p} \zeta_{i}\right)
$$

The bunch wakefield potential corresponding to this distribution is given by the Green's function for the 2D wakefield equation:

$$
\Phi_{B}(x, \zeta)=\Phi_{0} \frac{\eta}{N} \sum_{\zeta_{i}>\zeta} \sin \left(k_{p} \zeta-k_{p} \zeta_{i}\right) e^{-k_{p}\left|x-x_{i}\right|}
$$

The constant $\eta$ is proportional to the total bunch charge. We call this number the beam loading fraction, because, roughly speaking, $\eta=1$ corresponds to the beam loading limit. From the bunch wakefield (5) it is seen that $\eta$ can be interpreted as the ratio of amplitudes of the bunch wakefield and the laser wakefield. Note that the condition $\zeta_{i}>\zeta$ is due to causality, since there can be no wakefield in front of the source.

A particle tracking code has been written to perform simulations of bunch dynamics. This code solves Eq. (2) with a finite difference implementation and uses (3) and (5) as a description for the wakefields. In order to illustrate the mechanism of emittance growth as clearly as possible, the bunch initial conditions are chosen as follows: at injection, all particles have $x=x_{0}, P_{x}=0, P_{z}=P_{z 0}$, distributed uniformly in $\zeta$ between $\zeta_{0} \pm L$. For the simulation results shown here, we have used $P_{z 0}=20 m c, k_{p} L=0.3$.

Simulation results with variable bunch charge and radial offset $x_{0}$ are shown in Figs. 2-5. As an illustration, snap-

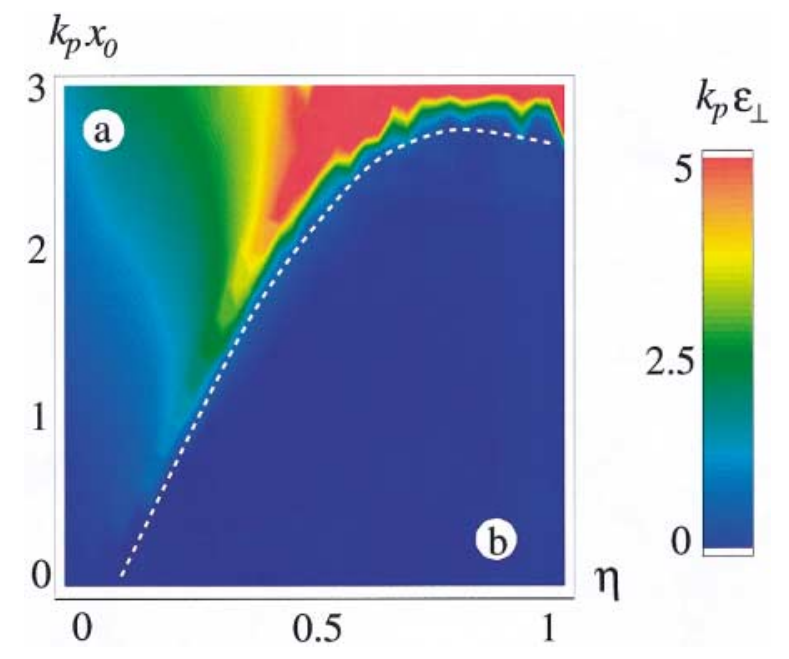

FIG. 2 (color). Contour plot of normalized transverse emittance $\epsilon_{\perp}$ after acceleration distance $L=1500 \lambda_{p}$ as a function of $x_{0}$ and $\eta$.

shots of $\left(\zeta, x, P_{x}\right)$-space and $\left(\zeta, P_{z}\right)$-space for two specific cases are shown in Fig. 6 . The parameters for these cases are $k_{p} x_{0}=2, \eta=0.4$ (case I) and $k_{p} x_{0}=2, \eta=0.8$ (case II).

In the plot of normalized transverse emittance (Fig. 2) two regions, denoted $a$ and $b$, can be clearly distinguished. To guide the eye, the boundary between these regions is indicated by a dashed curve. For comparison, this curve is shown in Figs. 3-5 as well. Region $a$ at low $\eta$ and high $x_{0}$ is a regime of large emittance growth, in which the electron bunch is subject to beam breakup. This can be seen from the typical spiral structure in $\left(\zeta, x, P_{x}\right)$-space (see Fig. 6 , case I). Region $b$ at high $\eta$ and low $x_{0}$ is a regime with much less emittance growth. We call this regime the selffocusing regime, because, as will be shown later, the selffocusing effect of the bunch wakefields is so strong that the electrons in the trailing part of the bunch are forced to

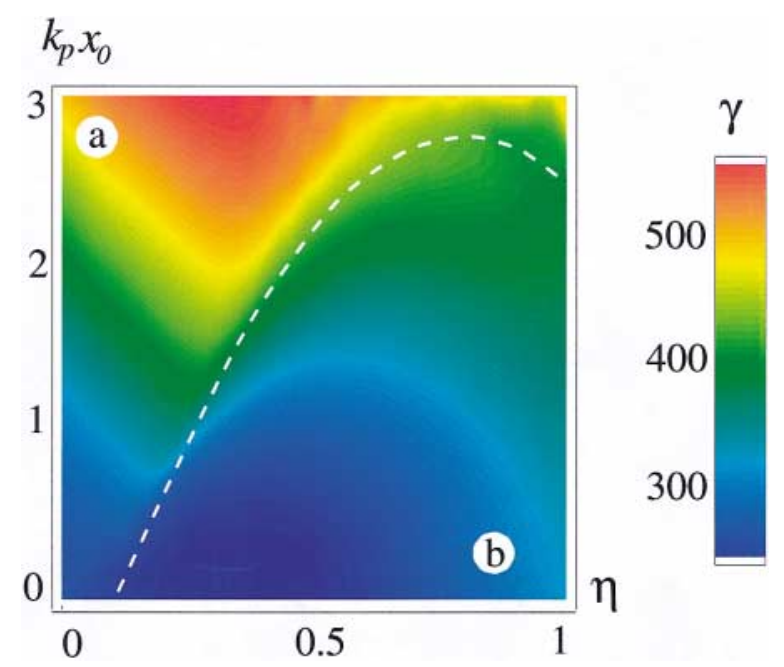

FIG. 3 (color). Contour plot of average energy $\gamma$ after acceleration distance $L=1500 \lambda_{p}$ as a function of $x_{0}$ and $\eta$. 


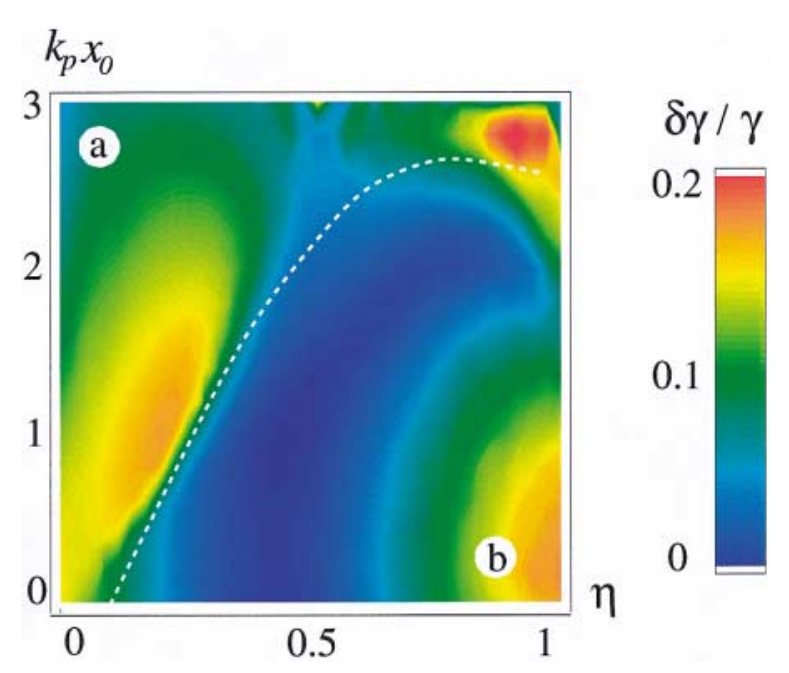

FIG. 4 (color). Contour plot of relative energy spread $\delta \gamma / \gamma$ after acceleration distance $L=1500 \lambda_{p}$ as a function of $x_{0}$ and $\eta$.

follow the electrons in the leading part. In $\left(\zeta, x, P_{x}\right)$-space (see Fig. 6, case II), the distribution is almost a line, which corresponds to a very small transverse emittance. From the dashed curve in Fig. 2 it is seen that the threshold value of $\eta$ for the self-focusing regime increases with increasing radial offset $x_{0}$. For values of $k_{p} x_{0}$ larger than about 2.6, there is no transition to the self-focusing regime.

The plot of average energy (Fig. 3) shows that the energy in the self-focusing regime is lower than in the beam breakup regime. This difference is explained by the radial dependence of the bunch wakefield. In the self-focusing regime, all bunch particles are around the same radial position, so they are at the maximum of the decelerating bunch wakefield, as can be seen from the factor $e^{-k_{p}\left|x-x_{i}\right|}$ in Eq. (5), which has its maximum at $\left|x-x_{i}\right|=0$. In the beam breakup regime the particles spread out radially, so

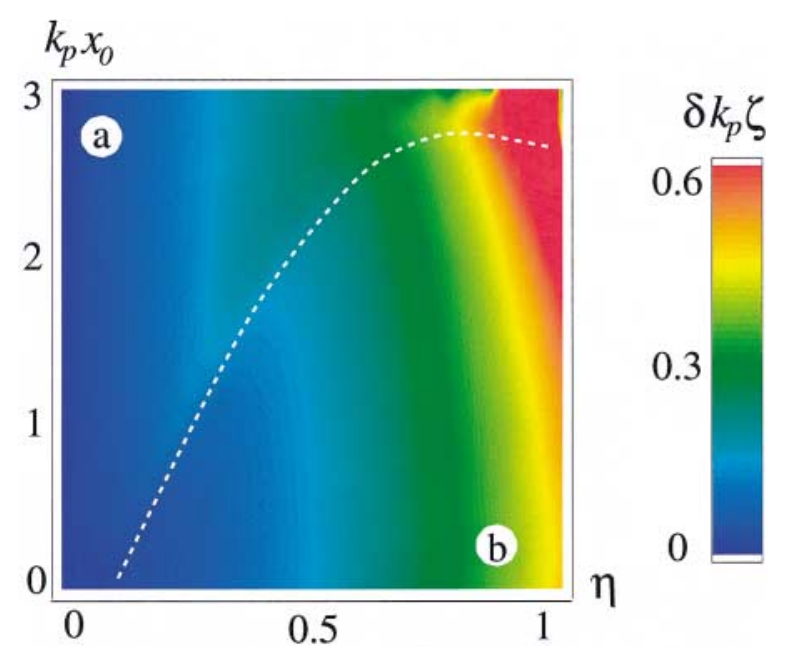

FIG. 5 (color). Contour plot of rms phase spread $\delta \zeta$ after acceleration distance $L=1500 \lambda_{p}$ as a function of $x_{0}$ and $\eta$. that the deceleration due to the bunch wakefields is less effective.

In the plot of relative energy spread (Fig. 4) a region of low energy spread is found in the self-focusing regime. The occurrence of low energy spread is a combination of bunch length and bunch wakefield effects [6]. Because of its phase dependence, the laser wakefield induces an energy spread in a bunch of finite length: in particular, it causes the trailing part of the bunch to gain more energy than the leading part. This is illustrated in the $\left(\zeta, P_{z}\right)$ snapshots of Fig. 6, case I. Because the bunch wakefield is decelerating, it induces the opposite effect, i.e., an energy loss of the trailing part of the bunch. Therefore, it is possible to minimize energy spread by proper phasing [5], so that both contributions to energy spread cancel each other after a particular acceleration distance. This is illustrated in the $\left(\zeta, P_{z}\right)$-snapshots of Fig. 6 , case II. Note especially snapshot 6(c), which has an almost flat bunch distribution.

The decelerating bunch wakefield also introduces an increase of bunch length, as illustrated in Fig. 5, which shows a clear trend of increasing phase spread with increasing $\eta$.
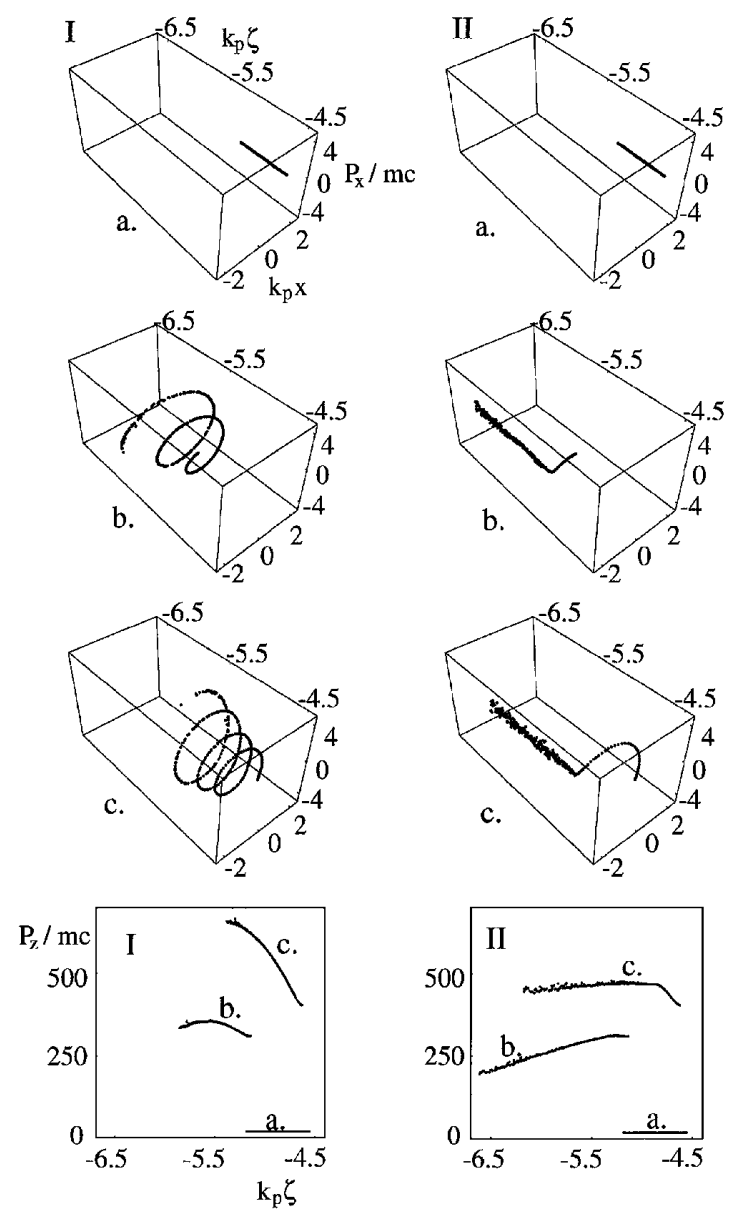

FIG. 6. Snapshots of $\left(\zeta, x, P_{x}\right)$-space (boxes) and $\left(\zeta, P_{z}\right)$ space (squares) with $\eta=0.4$ (I) and $\eta=0.8$ (II) at acceleration distances $L=0$ (a), $L=900 \lambda_{p}$ (b), and $L=1800 \lambda_{p}$ (c). 
In order to describe the difference in the transverse dynamics between the beam breakup regime and the selffocusing regime, we use a model in which the bunch is represented by $N$ particles, located at $\vec{r}_{\perp, i}$, with $\zeta_{N}<\ldots<$ $\zeta_{2}<\zeta_{1}$. The transverse equations of motion are

$$
\frac{d}{d t}\left(\gamma_{i} \frac{d}{d t} \vec{r}_{\perp, i}\right)+c^{2} \nabla_{\perp} \Phi\left(\vec{r}_{\perp, i}, \zeta_{i}\right)=0, \quad 1 \leq i \leq N
$$

Here $\gamma_{i}$ denotes the energy of the $i$ th particle. We assume that the transverse dynamics is much faster than the longitudinal dynamics, so we ignore the time dependence of all $\zeta_{i}$ and $\gamma_{i}$, where the bunch is assumed to be monoenergetic $\left(\gamma_{i}=\gamma, 1 \leq i \leq N\right)$. Approximating the laser wakefield in a Taylor series around the laser axis $\left(\vec{r}_{\perp}=0\right)$

$$
\Phi_{L}\left(\vec{r}_{\perp}, \zeta\right) \approx \Phi_{L 0}(\zeta)+\frac{1}{2} \vec{r}_{\perp}^{2} \Phi_{L 2}(\zeta)
$$

and the bunch wakefield in a Taylor series around the particles' transverse coordinates $\left(\vec{r}_{\perp}=\vec{r}_{\perp, i}\right)$

$$
\begin{aligned}
\Phi_{B}\left(\vec{r}_{\perp}, \zeta\right) \approx \sum_{\zeta_{i}>\zeta} & {\left[\Phi_{B 0}\left(\zeta, \zeta_{i}\right)+\frac{1}{2}\left(\vec{r}_{\perp}-\vec{r}_{\perp, i}\right)^{2}\right.} \\
& \left.\times \Phi_{B 2}\left(\zeta, \zeta_{i}\right)\right]
\end{aligned}
$$

we arrive at

$$
\frac{d^{2} \vec{r}_{\perp, i}}{d t^{2}}+\omega_{i}^{2} \vec{r}_{\perp, i}=\sum_{j<i} c_{j i}\left(\vec{r}_{\perp, j}-\vec{r}_{\perp, i}\right), \quad 1 \leq i \leq N
$$

Here $\omega_{i}^{2}=c^{2} \Phi_{L 2}\left(\zeta_{i}\right) / \gamma$ denotes the betatron frequency of the transverse oscillation of the $i$ th particle in the laser wakefield potential and $c_{j i}=c^{2} \Phi_{B 2}\left(\zeta_{j}, \zeta_{i}\right) / \gamma$ denotes the strength of the coupling of transverse oscillations of different particles induced by the self-focusing bunch wakefields. Because of the asymmetric interaction, the particle at $\zeta_{i}$ influences the particle at $\zeta_{j}$ for $j<i$ only. Equation (9) can be solved exactly with the following model for the betatron frequency and coupling parameters: $\omega_{i}^{2}=\omega_{0}^{2}-i \Delta, c_{j i}=(i-j) C$ if $i>j, c_{j i}=0$ otherwise. Assuming $\zeta_{i}=\zeta_{0}-i \delta$, the formula for $\omega_{i}^{2}$ can be understood as a Taylor expansion in $\zeta=\zeta_{0}$ for the betatron frequency. The coupling strength is proportional to the distance between the particles $(i-j)$ and to the bunch charge. With the initial conditions $\vec{r}_{\perp, i}(0)=\vec{r}_{0}$, $d \vec{r}_{\perp, i} / d t(0)=0(1 \leq i \leq N)$, and the definition $\hat{\omega}_{i}^{2}=$ $\omega_{i}^{2}+\sum_{j<i} c_{j i}$, we can write the solution to the system (9) in the form $\vec{r}_{\perp, i}=\vec{r}_{0} \sum_{j \leq i} a_{j i} \cos \left(\hat{\omega}_{i} t\right)$, where the coefficients $a_{j i}$ are given by

$$
\begin{gathered}
a_{j i}=\frac{C \Delta}{(\Delta-j C)(\Delta-j C+C)}, \quad j<i, \\
a_{i i}=\frac{\Delta}{\Delta-i C+C} .
\end{gathered}
$$

Since the parameter $C$ in our model is proportional to the bunch charge, the behavior of emittance as a function of $\eta$ (see Fig. 2) can be explained qualitatively from the behavior of (10) as a function of $C$. In the absence of coupling $(C=0)$, the solution is $\vec{r}_{\perp, i}(t)=\vec{r}_{0} \cos \left(\omega_{i} t\right)$, as expected. As $C$ increases, singularities at $C / \Delta=1 / N, \ldots, 1 / 2,1$ are encountered. A singularity occurs if $\hat{\omega}_{i}=\hat{\omega}_{j}$ for some $i>j$, namely if the transverse oscillation of the particle at $\zeta_{i}$ drives the transverse oscillation of the particle at $\zeta_{j}$ exactly in resonance. These resonances lead to emittance growth and beam breakup, as found in region $a$ of Fig. 2 . In the limit of strong coupling $(C \gg \Delta)$ the bunch behaves like a rigid rotator (the same oscillation for all particles), which can be seen from the estimate

$$
\left[\vec{r}_{\perp, i+1}(t)-\vec{r}_{\perp, i}(t)\right]^{2} \leq \vec{r}_{0}^{2}\left(\frac{\Delta}{\Delta-i C}\right)^{2}
$$

Physically, this means that the bunch electrons are trapped in a narrow potential well created by the strong focusing bunch wakefield. In its turn, this well oscillates in the potential well created by the laser wakefield. If the bunch is kept together by the strong self-focusing forces, there is much less emittance growth, as found in region $b$ of Fig. 2.

In conclusion, we have described a new regime of laser wakefield acceleration of an injected electron bunch with strong bunch wakefields. We call this regime the self-focusing regime, because of the strong self-focusing forces that reduce the emittance growth arising from misalignment errors. We have also shown that it is possible to reduce the energy spread by exploiting phase slippage effects.

[1] T. Tajima and J. Dawson, Phys. Rev. Lett. 43, 267 (1979).

[2] A. Modena et al., Nature (London) 377, 606 (1995).

[3] S. Chen et al., Phys. Plasmas 6, 4739 (1999).

[4] T. Katsouleas et al., Part. Accel. 22, 81 (1987).

[5] T. Chiou and T. Katsouleas, Phys. Rev. Lett. 81, 3411 (1998).

[6] A. Reitsma, R. Trines, and V. Goloviznin, IEEE Trans. Plasma Sci. 26, 1150 (2000).

[7] T. Katsouleas et al., AIP Conf. Proc. 395, 75 (1996).

[8] A. Reitsma et al., Phys. Rev. E 63, 046502 (2001).

[9] S. Cheshkov et al., Phys. Rev. ST Accel. Beams 3, 071301 (2000).

[10] C. Chiu, T. Tajima, and S. Cheshkov, Phys. Rev. ST Accel. Beams 3, 101301 (2000).

[11] C. Schroeder, D. Whittum, and J. Wurtele, Phys. Rev. Lett. 82, 1177 (1999).

[12] P. Chen, Part. Accel. 20, 171 (1987).

[13] W. Panofsky and W. Wenzel, Rev. Sci. Instrum. 27, 967 (1956). 